\title{
Kinetics of photoinduced surface patterning in chalcogenide thin films
}

\author{
Yu. Kaganovskii, ${ }^{1, a)}$ D. L. Beke, ${ }^{2}$ and S. Kökényesi ${ }^{3}$ \\ ${ }^{1}$ Department of Physics, Bar-Ilan University, Ramat-Gan 52900, Israel \\ ${ }^{2}$ Department of Solid State Physics, University of Debrecen, H-4010 Debrecen, Hungary \\ ${ }^{3}$ Department of Experimental Physics, University of Debrecen, H-4010 Debrecen, Hungary
}

(Received 6 May 2010; accepted 19 July 2010; published online 10 August 2010)

\begin{abstract}
The kinetics of photoinduced variations in surface profile in chalcogenide glass films under illumination is described. It is demonstrated that the competition between the stress-induced atomic flux (toward irradiated regions of the film) and the diffusion flux induced by an increase in the bulk energy due to broken bonds (and directed from irradiated to dark regions) can result in either a positive or negative net mass transfer in the irradiated region. Depending on the light intensity, one can obtain either formation of bumps or depressions in the illuminated regions. (C) 2010 American Institute of Physics. [doi:10.1063/1.3477957]
\end{abstract}

There is high basic and technological interest for chalcogenide glasses (ChGs) (Refs. 1-6) (e.g., $\mathrm{As}_{c} \mathrm{~S}_{1-c}$ or $\left.\mathrm{As}_{c} \mathrm{Se}_{1-c}\right)$, since a rich variety of light induced structural changes can be produced in them. Irradiation by near bandgap light evokes formation of nonequilibrium carriers and after their nonradiative relaxation can lead to formation of broken chemical bonds, can induce giant expansion, ${ }^{7}$ and increased fluidity. ${ }^{8}$ Besides the expansion, resulting in convexity of surface profile in irradiated regions, a photoinduced mass transfer, directed from illuminated to dark areas of ChG films was detected at high enough light intensities. The mass transfer observed is not the result of warming up caused by light absorption but it is a light-induced (ballistic) mass transfer. Indeed, under illumination by a polarized light, the mass transfer occurs only in the directions parallel to $E$-vector. 5,9 Under homogeneous illumination, the scratches on the film surface directed perpendicularly to $E$-vector of the polarized light, were flattened and cracks developed, ${ }^{9}$ quite similar to their thermal evolution caused by capillary forces, ${ }^{10}$ whereas the scratches and cracks parallel to $E$-vector left without changes. Thus, the photoinduced diffusion coefficients of the glass constituents in the direction parallel to the $E$-vector, $D_{x x}$, exceeded several times those in perpendicular direction, $D_{y y}$.

In spite of multiple, sometimes controversial experimental results, the driving forces, and the kinetics of these processes are still not clear.

It is known that ChGs have relatively low photoluminescence efficiency. This means that after excitation by light with appropriate photon energy, a nonradiative recombination of electron-hole pairs occurs, which is accompanied by dissipation of rather large energy. ${ }^{11}$ This recombination may occur via a transient exciton, which can be visualized as a transient intimate valence alternation pair. ${ }^{12,13}$ The changes in the local charge or valence state, and thus perturbations of the covalent coordination, are quite localized. The local deformation of bonds can lead, after recombination, to photoinduced changes in bonding configuration, ${ }^{14}$ so that atoms can move over atomic distances in the local volume where the recombination energy was released. Thus, we can asso-

\footnotetext{
a) Author to whom correspondence should be addressed. Electronic mail: kagany@mail.biu.ac.il. Tel.: 972-3-531-7864. FAX: 972-3-738-4054.
}

ciate the elementary diffusion jump with the recombination event, and jumps of both pnictide $(P)$ and chalcogene $(C)$ atoms depend on their local configurations. As transient excitons possess a dipole moment, one can expect that preferable orientation of alternation pairs, as well as atomic jumps, will be parallel to the polarization plane.

Consider a $\mathrm{ChG}$ film with thickness $H$ on transparent substrate illuminated by polarized light with the intensity distribution $I(x)$; the $x$-axis is parallel to polarization direction. Under inhomogeneous illumination, the photoinduced expansion becomes also inhomogeneous. This results in formation of surface profile and creates lateral compressive stresses in the illuminated regions. The chemical potentials of $P$ and $C$ atoms depend on coordinates and can be written as follows:

$$
\begin{aligned}
\mu_{k}(x)= & \mu_{0 k}+\varepsilon_{k} \bar{\omega} n_{e x}(x)+K(x) \bar{\omega} \gamma(x)+\sigma(x) \Delta \bar{\omega}(x), \\
& k=P, C .
\end{aligned}
$$

Here, $\mu_{0 k}$ is the bulk chemical potential of the atoms without illumination, $\varepsilon_{k}$ is the increase in the energy of appropriate chemical bonds due to their deformation around the excitons, $n_{e x}$ is the number of transient excitons per unit volume, and $\bar{\omega}$ is the average atomic volume. The term $K \gamma \omega$ gives an account for capillary forces caused by the curvature of the surface profile $z(x, t), K(x) \approx-z_{x x}^{\prime \prime}$ is the local surface curvature (for small surface slopes), $\gamma$ is the surface tension, $\sigma$ is the stress caused by the photoinduced volume expansion of the film, $\Delta \bar{\omega}$ is the average volume expansion per atom, and $n_{e x}, \Delta \bar{\omega}$, and $\sigma$ are functions of the light intensity and thus functions of $x$.

Under cw illumination, in steady sate, the number of transient excitons, $n_{e x}$, can be estimated as

$$
n_{e x}=\alpha I \tau / \varepsilon_{g} .
$$

Here, $\alpha$ is the light absorption coefficient, $I$ is the light intensity, $\varepsilon_{g}$ is the photon energy, and $\tau$ is the average exciton lifetime. The term $\alpha \cdot I / \varepsilon_{g}$ gives the number of photons absorbed in unit volume per unit time, which is approximately the number of excited electron-hole pairs transformed into localized excitons. Then the term $\varepsilon_{k} \bar{\omega} n_{e x}(x)$ in Eq. (1) can be rewritten as $q_{k} I(x)$ with 


$$
q_{k} \approx \alpha \tau \bar{\omega} \varepsilon_{k} / \varepsilon_{g} .
$$

Substituting $\alpha=5 \times 10^{4} \mathrm{~cm}^{-1}, \varepsilon_{k} / \varepsilon \approx 0.5, \tau \approx 10-100 \mathrm{~ns},{ }^{15}$ and $\bar{\omega} \approx 5 \times 10^{-23} \mathrm{~cm}^{3}$ into Eq. (3) we have $q_{k}$ $\approx 10^{-25}-10^{-26} \mathrm{~cm}^{2} \mathrm{~s}$.

The lateral compressive stress, $\sigma$, in the last term of Eq. (1), is proportional to the relative linear expansion, $\Delta a / a$ $\approx \Delta \bar{\omega} / 3 \bar{\omega}$, i.e., $\sigma \approx E_{Y} \Delta \bar{\omega} / 3 \bar{\omega}\left(E_{Y}\right.$ is the elastic modulus). Following the experimental data of Ref. 7, one can describe the intensity dependence of the expansion by the following empirical formula:

$$
\frac{\Delta \bar{\omega}}{\bar{\omega}} \approx \frac{\beta I e_{m}}{\beta I+e_{m}},
$$

where $\beta$ is a coefficient, which can be estimated from the data on photoinduced volume expansion, $e_{m}$ is the maximal (saturation) deformation.

The second term in Eq. (1), $q_{k} I$, initiates mass flow from light to dark regions and competes with the last term $\sigma \Delta \bar{\omega}$, caused by lateral compressive stresses. The expansion is possible only in $z$-direction; the lateral volume expansion is hindered by the not illuminated regions. The stress induced mass flow is oriented toward the illuminated regions, resulting in relaxation of the stresses and formation of ridges in the illuminated areas.

The light induced local gradients of $\mu_{k}$ evoke lateral diffusion fluxes of $P$ and $C$ atoms

$$
J_{k x}(x)=-\frac{D_{k}(x)}{k T} N_{k} \frac{\partial \mu_{k}(x)}{\partial x},
$$

as well as stress-induced fluxes in $z$-direction, toward the free surface of the film. These fluxes also depend on $x$ as follows:

$$
J_{k z}(x)=-\frac{D_{k}(x)}{k T} N_{k} \frac{\partial \mu_{k}(x)}{\partial z} .
$$

Here, $N_{k}$ is the number of $P$ and $C$ atoms per unit volume, and $D_{k}$ is the appropriate ballistic ${ }^{16}$ diffusion coefficient, which we assumed to be proportional to the light intensity; $D_{k}=\delta_{k} I$. We consider bulk diffusion as the main mechanism of diffusion flow and neglect surface diffusion mechanism. Indeed, according to data on spectral dependence of photoinduced viscosity, ${ }^{17,18}$ light with photon energy higher than the band gap, does not induce mass transfer, although electron-hole pairs are excited in the subsurface layer and may induce fast surface diffusion.

The variation in the surface profile is given by

$$
\frac{\partial z}{\partial t}=-H\left(\frac{\partial J_{P x}}{\partial x} \omega_{P}+\frac{\partial J_{C x}}{\partial x} \omega_{C}\right)+J_{P z}(x) \omega_{P}+J_{C z}(x) \omega_{C} .
$$

Due to small film thickness (compared to $1 / \alpha$ ), we can assume that diffusion coefficients depend only on $x$-coordinate and $-\partial \mu_{k}(x) / \partial z \approx \sigma(x) \Delta \bar{\omega}(x) / H$.

The equation for the evolution of the surface profile, $z(x, t)$ is rather complicated. Thus we solved the problem numerically. The values of parameters used for our calculations are presented in Table I.

We assumed independent diffusion fluxes of pnictides and chalcogenes and used the effective diffusion coefficient
TABLE I. Parameters used for numerical calculations.

\begin{tabular}{ccccccc}
\hline \hline $\begin{array}{c}\bar{\omega} \\
\left(\mathrm{cm}^{3}\right)\end{array}$ & $\begin{array}{c}\gamma \\
\left(\mathrm{erg} / \mathrm{cm}^{2}\right)\end{array}$ & $\begin{array}{c}E_{Y} \\
\left(\mathrm{dyn} / \mathrm{cm}^{2}\right)\end{array}$ & $\begin{array}{c}\beta \\
\left(\mathrm{cm}^{2} \mathrm{~s} / \mathrm{erg}\right)\end{array}$ & $\begin{array}{c}\alpha \\
\left(\mathrm{cm}^{-1}\right)\end{array}$ & $\begin{array}{c}e_{m} \\
(\mu \mathrm{m})\end{array}$ & $\begin{array}{c}H \\
5 \times 10^{-23}\end{array}$ \\
\hline $5 \times 10^{2}$ & $2 \times 10^{11}$ & $10^{-10}-10^{-9}$ & $5 \times 10^{4}$ & $0.01-0.02$ & 2 \\
\hline \hline
\end{tabular}

$D=D_{P} c+D_{C}(1-c)=\delta \cdot I$.

Thus, we neglected the segregation caused by the difference of pnictide and chalcogene diffusion fluxes. The coefficient $\delta$ was used as a fitting parameter.

We considered periodic light intensity distributions created by interference of two crossed coherent beams and used for recording optical gratings as follows:

$$
I(x)=2 I_{0}\left(1+\cos \frac{2 \pi x}{\Lambda}\right) .
$$

Here, $I_{0}$ is the intensity of each of the beams and $\Lambda$ is the period of the intensity oscillation.

We assumed, as the first approximation, that the shape of the surface profile formed under illumination varies as

$$
z(x, t)=h(t) \cos \frac{2 \pi x}{\Lambda}
$$

where $h(t)$ can be positive or negative depending on the direction of the mass flow, it defines maximum height or depth of the profile as a function of exposure time.

Substitution of Eq. (10) in Eq. (7) yields equation for $h(t)$ as follows:

$$
\frac{d h(t)}{d t}=A h(t)+B .
$$

Here, $A$ and $B$ are coefficients, which depend on the $x$-coordinate caused by the dependence $I(x)$ on $\delta$ and other parameters of Table I. Solution of Eq. (11) for the initial condition $h(0)=h_{0}$ is

$$
h(t)=-\frac{B}{A}+\frac{\left(A h_{0}+B\right) \exp (A t)}{A} .
$$

Depending on the sign of the coefficients $A$ and $B, h(t)$ will be positive or negative. Accordingly, the variation in the light intensity (with appropriate set of parameters) results in inversion of the direction of the resultant diffusion flow.

Indeed, it can be seen in Fig. 1 that, if we start from a flat profile $\left(h_{0}=0\right)$, at low intensities there is a growth of hillock (bump) $[h(t)>0]$, whereas at higher intensities there is a depression $[h(t)<0]$. The threshold intensity depends on $\Lambda$ and on the maximal deformation $e_{m}$.

Similar result was obtained when we considered Gaussian intensity distribution.

The rate of the mass transfer, $d h / d t$, essentially depends on $I$, as well as on $\Lambda$. The height of the bump and the depth of the dip, as well as the amplitude of periodic profile, are stabilized with time by capillary forces. Due to this stabilization, maximum amplitudes of the forming gratings rapidly grow with the grating period, in agreement with experiments. $^{5}$

We have found rather good agreement between our calculations and experimental data on the bump growth ${ }^{6}$ at low intensities $\left(I_{0}=0.35 \mathrm{~W} / \mathrm{cm}^{2}\right)$ during recording of grating 

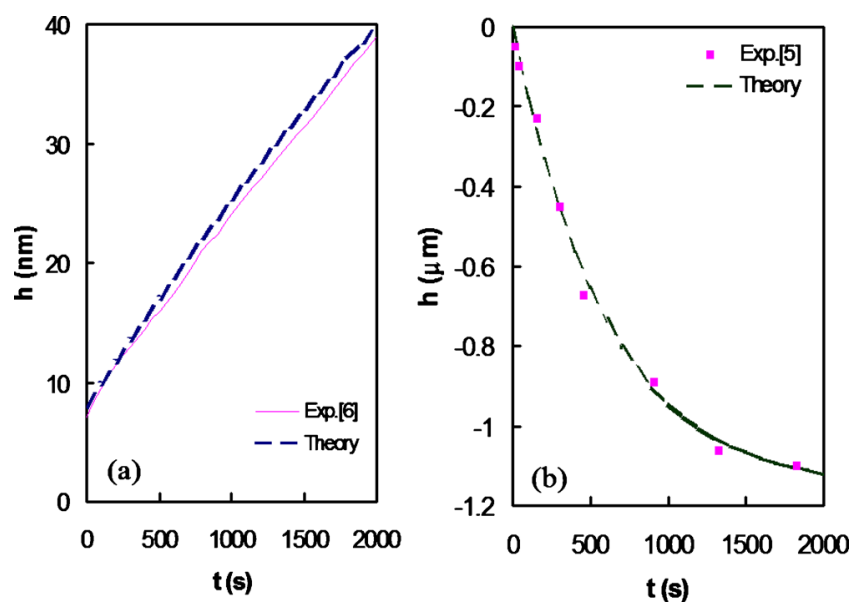

FIG. 1. (Color online) Kinetics of bump growth (a) or depression formation (b) under illumination by two crossing beams of low $\left(I_{0}=0.35 \mathrm{~W} / \mathrm{cm}^{2}\right)$ and higher intensity $\left(I_{0}=52.5 \mathrm{~W} / \mathrm{cm}^{2}\right)$, respectively. Comparison of the theory (dotted lines) with the experimental data taken from Ref. 6 (a) and Ref. 5 (b).

with the period $\Lambda=3.6 \mu \mathrm{m}$ in the film $\mathrm{As}_{30} \mathrm{Se}_{70}$ [Fig. 1(a)] and on the depression formation at higher intensities ${ }^{5}$ $\left(I_{0}=52.5 \mathrm{~W} / \mathrm{cm}^{2}\right)$ during recording of $\Lambda=13.5 \mu \mathrm{m}$ grating [Fig. 1(b)]. We have found in both cases $\delta \approx 1.2$ $\times 10^{-19} \mathrm{~cm}^{4} / \mathrm{erg}$ that corresponds to variation $D$ in the range $1.2 \times 10^{-12}-2.4 \times 10^{-10} \mathrm{~cm}^{2} / \mathrm{s}$ for $1<I<200 \mathrm{~W} / \mathrm{cm}^{2}$.

In summary, there are two main competitive fluxes induced by inhomogeneous illumination of ChG films. One is caused by photoinduced stress relaxation and directed toward illuminated surface; the second is determined by gradients of bulk free energy caused by broken and deformed chemical bonds and directed toward dark regions. The direction of the resultant mass transfer depends on the light intensity and results in bump formation at low intensities and dip formation at higher intensities. The kinetics of the mass transfer is described by photoinduced (ballistic) bulk diffusion coefficient, which is proportional to the light intensity.

The authors are thankful to V. B. Sandomirsky and M. I. Klinger for useful discussions. This work was supported by the Grant Nos. K67685 and CK80126 of the Hungarian Scientific Research Fund. The work is also supported by TÁMOP 4.2.1-08/1-2008-003 project.

${ }^{1}$ S. R. Elliott, J. Non-Cryst. Solids 81, 71 (1986).

${ }^{2}$ A. Ozols, O. Salminen, P. Riihola, and P. Monkkonen, J. Appl. Phys. 79, 3397 (1996).

${ }^{3}$ H. Hisakuni and K. Tanaka, Science 270, 974 (1995).

${ }^{4}$ K. Tanaka and H. Hisakuni, J. Non-Cryst. Solids 198-200, 714 (1996).

${ }^{5}$ A. Saliminia, T. V. Galstian, and A. Villeneuve, Phys. Rev. Lett. 85, 4112 (2000).

${ }^{6}$ M. L. Trunov, P. M. Lytvyn, P. M. Nagy, and O. M. Dyachyns'ka, Appl. Phys. Lett. 96, 111908 (2010).

${ }^{7}$ H. Hisakuni and K. Tanaka, Appl. Phys. Lett. 65, 2925 (1994).

${ }^{8}$ D. T. Kastrissios and N. Yannopoulos, J. Non-Cryst. Solids 299-302, 935 (2002).

${ }^{9}$ M. L. Trunov, J. Phys. D: Appl. Phys. 41, 074011 (2008).

${ }^{10}$ W. W. Mullins, J. Appl. Phys. 30, 77 (1959).

${ }^{11}$ R. A. Street, Solid State Commun. 24, 363 (1977).

${ }^{12}$ M. Kastner, D. Adler, and H. Fritzsche, Phys. Rev. Lett. 37, 1504 (1976).

${ }^{13}$ M. Kastner and H. Fritzsche, Philos. Mag. B 37, 199 (1978).

${ }^{14}$ H. Fritzsche, Semiconductors 32, 850 (1998).

${ }^{15}$ J. Singh, J. Optoelectron. Adv. Mater. 7, 129 (2005).

${ }^{16}$ G. Martin, Phys. Rev. B 30, 1424 (1984).

${ }^{17}$ D. K. Tagantsev and S. V. Nemilov, Fiz. Khim. Stekla 15, 397 (1989).

${ }^{18}$ K. Tanaka, N. Terakado, and A. Saitoh, J. Optoelectron. Adv. Mater. 10, 124 (2008). 